\title{
Development of Exhaled Breath Diagnosis Sensors for Rapid Identification of COVID-19 Patients
}

\author{
Cuili Xue ${ }^{1}$, Amin Zhang ${ }^{1}$, Yunsheng Chen ${ }^{2}$, Hui Liang ${ }^{3}$, Jing Tian ${ }^{3}$, Jingpu Zhang ${ }^{4}$, Chen Zhou ${ }^{3}$, \\ Jian $\mathrm{Ni}^{1,3}$, Han $\mathrm{Jin}^{1,3}$, Daxiang Cui ${ }^{1,3}$ \\ ${ }^{1}$ Institute of Nano Biomedicine and Engineering, Shanghai Engineering Center for Intelligent Diagnosis and Treatment Instrument, \\ Department of Instrument Science and Engineering, School of Electronic Information and Electrical Engineering, Shanghai Jiao Tong \\ University, 800 Dongchuan Road, Shanghai 200240, China. \\ ${ }^{2}$ Department of Plastic and Reconstructive Surgery, Shanghai Ninth People's Hospital, School of Medicine Shanghai Jiao Tong \\ University, 639 Zhizaoju Road, Shanghai 200011, China. \\ ${ }^{3}$ National Engineering Research Center for Nanotechnology, 28 Jiangchuan Road, Shanghai 200241, China. \\ ${ }^{4}$ Shanghai Public Health Clinical Center, Fudan University, 2901 Caolang Road, Jinshan District, Shanghai 201508, China.
}

Corresponding authors. E-mail: hanjin10@sjtu.edu.cn; dxcui@sjtu.edu.cn

Received: Feb. 23, 202I; Accepted: Aug. 5, 202I; Published: Aug. 6, 202I

Citation: CuiliXue, Amin Zhang, Yunsheng Chen, Hui Liang, Jing Tian, Jingpu Zhang, Chen Zhou, Jian Ni, Han Jin, and Daxiang Cui, Development of Exhaled Breath Diagnosis Sensors for Rapid Identification of COVID-19 Patients. Nano Biomed. Eng., 202I, 13(3): 225-228.

DOI: $10.5101 /$ nbe.v13i3.p225-228.

\begin{abstract}
The novel coronavirus pneumonia, a global pandemic disease named as coronavirus disease 2019, has caused enormous losses on the health and economies of people all over the world, while there is still a lack of quick and sensitive diagnostic method and effective therapy. Developing rapid diagnostic method for coronavirus disease 2019 has become exceptional urgent. Herein we report a rapid diagnostic method for the novel coronavirus through monitoring the volatile biomarkers in human exhaled breath. The breath volatile biomarkers are derived from the metabolism of novel coronavirus, including acetoin, 2,4,6-trimethylpyridine, 3-methyl tridecane, tetradecane, isooctyl alcohol, pentadecane, hexadecane, 1-methylene- $1 \mathrm{H}$-indene. By comparing the types and concentrations of the volatile biomarkers in human exhaled breath combined with SERS sensor, we could distinguish between the healthy person and the patients with coronavirus disease 2019. This work confirms that various volatile organic compounds metabolized by novel coronavirus can be employed for rapidly screening of patients with coronavirus disease 2019, and has broad application prospects in the prevention and control of the epidemic.
\end{abstract}

$\overline{\text { Keywords: Coronavirus disease 2019, Volatile organic compounds, Exhaled breath diagnosis, Sensor }}$

The coronavirus disease 2019(COVID-19) is one kind of acute infectious disease caused by the novel coronavirus [1, 2]. The main symptoms of COVID-19 infection include respiratory symptoms such as fever, cough, shortness of breath, and breath difficulty. In some severe cases, the infection can lead to pneumonia, severe acute respiratory syndrome, kidney failure, and even death. Up to date, due to the lacking of effective antiviral drugs to treat COVID-19 virus, the isolation and supportive treatment are still the main approaches in controlling COVID-19 spread. Therefore, rapid screening and effective isolation remain the optimal ways to defeat the new coronavirus for those who are susceptible to COVID-19 [3]. So far, the main diagnosis techniques are based on COVID-19 virus nucleic acid and antibody, but there are still some demerits such as difficulty in samples collection, long detection time, and false positive or negative detection 
results. Therefore, it is extremely practical and urgent to develop a rapid diagnostic method for COVID-19 [4, 5].

In ancient Greece, residents used their noses to identify special smells in the patient's breath to diagnose certain diseases [6, 7]. In the 18th century, Antoine Lavoisier, a pioneer of modern chemical analysis, first finished the analysis of exhaled gases and discovered that the exhaled gases contained $\mathrm{CO}_{2}$, opening up a new field in the analysis of exhaled gases. Since then, researchers have shown great interest in analyzing human exhaled breath. With the development of gas chromatography (GC) technology, Nobel Laureate Linus Pauling discovered that human exhaled breath contained more than 200 volatile organic compounds with the concentrations ranging from picomoles per liter $(\mathrm{pM})$ to micromole per liter $(\mu \mathrm{M})[8$, 9], opening a new «window» for researchers to study human metabolism. With the continuous development of analytical technologies, researchers have carried out detailed studies on human exhaled breath and finally make a conclusion that the components of human exhalation are complex and may be classified into inorganic gases, volatile organic compounds (VOCs) and non-volatile substances [8, 10-13]. With the indepth study of the composition in human exhaled breath [14-18], researchers have gradually discovered that the types and concentrations of VOCs in human breath are closely related to some diseases of the human body. Therefore, numerous studies have been conducted to screen out different biomarkers in exhaled breath between healthy people and patients were done, and researchers have established several breath analysis methods for the diagnosis of disease. Breath analysis technology has obvious advantages in disease diagnosis, such as non-invasive technique without imposing any physical or psychological burden on the patient and no interference in human breath samples. Nowadays, as a new disease diagnosis method, breath analysis has aroused more attention, and have been widely used in the screening tumors or metabolism diseases [19-24].

The VOCs in exhaled breath can reflect the metabolic status of the human body, providing a new diagnosing strategy in monitoring physiological and biochemical changes of the human body. Furthermore, breath analysis is particularly suitable for diagnosing long-term diseases. Under the condition of stable exhalation composition, accurate diagnosis of diseases could be achieved.

As shown in Table 1 , the selected exhaled VOCs markers are derived from the metabolism of novel coronavirus, mainly including styrene, acetoin, 2,4,6-trimethylpyridine, 3-methyl tridecane, tetradecane, isooctyl alcohol, pentadecane, hexadecane, 1-methylene-1H-indene.

Table 1 The VOCs biomarkers to distinguish COVID-19 patients from healthy persons

\begin{tabular}{cc}
\hline No. & Biomarkers \\
\hline 1 & Styrene \\
2 & Acetoin \\
3 & 2,4,6-Trimethylpyridine \\
4 & 3-Methyl tridecane \\
5 & Tetradecane \\
6 & Isooctyl alcohol \\
7 & Pentadecane \\
8 & Hexadecane \\
9 & 1-Methylene-1H-indene \\
\hline
\end{tabular}

The VOCs were screened out by gas chromatography from the metabolic gas of human lung fibroblasts, which were co-cultured with inactivated new coronavirus. Moreover, the normally cultured human lung fibroblast metabolites were used as a control. The detailed operation procedures showed as following: the collected novel coronavirus culture medium samples were injected by a headspace sampling device, concentrated by a solid phase microextraction device, and finally sent to the gas chromatographymass spectrometer to detect the content of VOSs. After comparing the gas chromatograms of experimental group (curve a in Fig. 1) and control group (curve b in Fig. 1), the obvious differences between these two spectrograms were found. Nine peaks labeled with

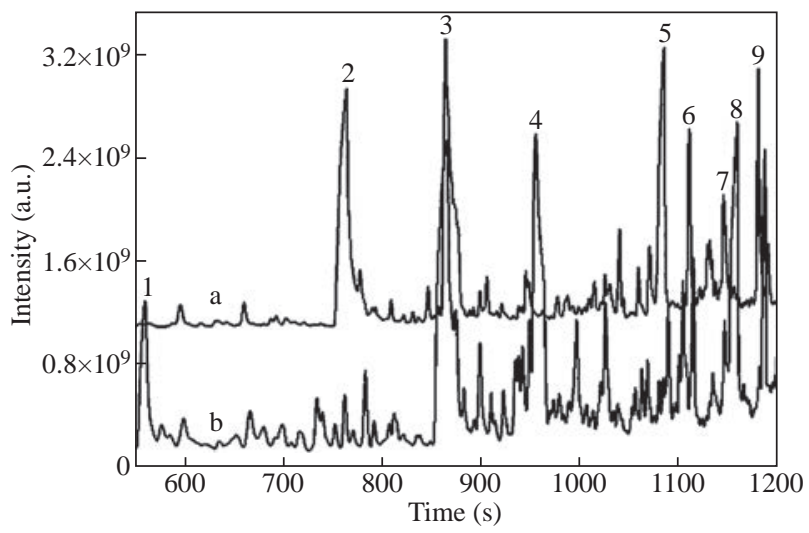

Fig. 1 Gas chromatogram of COVID-19 group (curve a) and health group (curve b), and the peak numbers corresponds to Table 1. 
numbers in gas chromatograms corresponded to the biomarkers in Table 1 and were selected for COVID-19 analysis.

In this work, we first screened out specific breath VOCs biomarkers associated with novel coronavirus infection via gas chromatography-mass spectrometry, then collected the breath gas samples from cured COVID-19 patients to verify the validity of markers, and finally established primary diagnosis standard to distinguish COVID-19 patients from healthy uninfected persons, which is as follows: those biomarkers such as 2,4,6-Trimethylpyridine, Tetradecan, 1-methylene-1H-indene and Acetoin are closely associated with COVID-19 infectious patients, which were used to identify COVID-19 patients, those biomarkers such as Isooctyl alcohol, Pentadecane, Styrene and 3-methyl tridecane are closely associated with healthy persons, which were used to identify uninfected healthy persons.

Further, we developed the SERS (Surface enhanced Raman scattering) sensors for ultrasensitive detection of nine exhale breath markers based on our previous reports [25-27]. So far, we are cooperating with Public Health Clinical Center, collecting the exhaled breath samples from COVID-19 patients, and verifying the coincidence of breath volatile markers-based diagnosis with $\mathrm{CT}$ images and nucleic acid test-based clinical diagnosis.

In summary, our primary study shows that human VOCs caused by novel coronavirus can be used as biomarkers for the breath diagnosis of COVID-19 patients, and SERS sensors combined with exhaled breath biomarkers have great application potential in COVID-19 patients screening. Further work will optimize this diagnostic system to improve the consistency between the breath volatile markers-based diagnosis and clinical diagnosis, and will translate this diagnosis system into clinical application in the near future.

\section{Acknowledgements}

This work was supported by Key Basic Research Program of China (No.2017YFA0205304 and 2015CB931802), Innovative Team Project of Nature Scientific Foundation of China (No.81921002), and Medical Engineering Cross Project of Shanghai Jiao Tong University (YG2017ZD12).

\section{Conflict of Interests}

The authors declare no conflict of interest.

\section{References}

[1] B.A. Resnick, P.C. Mui, J. Bowie, et al., The COVID-19 Pandemic: An Opportunity to Transform Higher Education in Public Health. Public Health Reports. 2021, 136(1): 23-26.

[2] A.B. Gussow N. Auslander, Y.I. Wolf, et al., Prediction of the incubation period for COVID-19 and future virus disease outbreaks. Bmc Biology, 2020, 18(1): 1-12.

[3] A. Higham, A. Mathioudakis, J. Vestbo, et al., COVID-19 and COPD: a narrative review of the basic science and clinical outcomes. European respiratory review, 2020, 29(158).

[4] A. Natarajan, H.W. Su, and C. Heneghan, Assessment of physiological signs associated with COVID-19 measured using wearable devices. Npj Digital Medicine, 2020, 3(1): $1-8$

[5] K.G. Aghila Rani, M.A. Hamad, D.M. Zaher, et al., Drug development post COVID-19 pandemic: toward a better system to meet current and future global health challenges. Expert opinion on drug discover, 2021, 16(4): 365-371.

[6] J.K. Schubert, W. Miekisch, K. Geiger, et al., Breath Analysis in Critically Ill Patients: Potential and Limitations. Expert Review of Molecular Diagnostics, 2004, 4(5): 619-629

[7] W.Q. Cao, Y.X. Duan. Breath analysis: Potential for clinical diagnosis and exposure assessment. Clinical Chemistry, 2006, 52(5): 800-811.

[8] N.J.W. Rattray, Z. Hamrang, D.K. Trivedi, et al., Taking your breath away: metabolomics breathes life in to personalized medicine. Trends Biotechnol, 2014, 32(10): 538-548.

[9] A.S. Modak, Breath biomarkers for personalized medicine. Pers Med, 2010, 7(6): 643-653.

[10] P.M. van Oort, P. Povoa, R. Schnabel, et al., The potential role of exhaled breath analysis in the diagnostic process of pneumonia-a systematic review. J Breath Res, 2018, 12(2): 024001.

[11] J. Pang, J. Feldman, V. Liberman, et al., Don't Waste Your Breath: A Study of Inhaler Disposal at Hospital Discharge for Copd Patients with Acute Exacerbations. Journal of General Internal Medicine, 2015, 30: 144.

[12] W. Miekisch, J.K. Schubert, and G.F. NoeldgeSchomburg, Diagnostic potential of breath analysis focus on volatile organic compounds. Clinica chimica acta, 2004, 347(1-2): 25-39.

[13] M. Alonso, J.M. Sanchez. Analytical challenges in breath analysis and its application to exposure monitoring. TracTrend Anal Chem, 2013, 44: 78-89.

[14] L. Fleming, D. Gibson, D. Hutson, et al., Breath emulator for simulation and modelling of expired tidal breath carbon dioxide characteristics. Computer methods and programs in biomedicine, 2020, 200: 105826.

[15] P.J. Chien, T. Suzuki, M. Ye, et al., Ultra-Sensitive Isopropanol Biochemical Gas Sensor (Bio-Sniffer) for Monitoring of Human Volatiles. Sensors, 2020, 20(23): 6827.

[16] L. Andre, N. Desbois, C.P. Gros, et al., Porous materials applied to biomarker sensing in exhaled breath for monitoring and detecting non-invasive pathologies. Dalton T. 2020, 49(43): 15161-15170.

[17] S.M. Aghaei, A. Aasi, S. Farhangdoust, et al., Graphene- 
like BC6N nanosheets are potential candidates for detection of volatile organic compounds (VOCs) in human breath: A DFT study. Appl Surf Sci, 2021, 536: 147756.

[18] A. Aasi, S.M. Aghaei, and B. Panchapakesan, A density functional theory study on the interaction of toluene with transition metal decorated carbon nanotubes: a promising platform for early detection of lung cancer from human breath. Nanotechnology, 2020, 31(41): 415707.

[19] V. Shestivska, K. Dryahina, J. Nunvar, et al., Quantitative analysis of volatile metabolites released in vitro by bacteria of the genus Stenotrophomonas for identification of breath biomarkers of respiratory infection in cystic fibrosis. J Breath Res, 2015, 9(2): 027104.

[20] M. Phillips, J.P. Boehmer, R.N. Cataneo, et al., Heart allograft rejection: Detection with breath alkanes in low levels (the HARDBALL study). J Heart Lung Transpl, 2004, 23: 701-708.

[21] B.J. Novak, D.R. Blake, S. Meinardi, et al., Exhaled methyl nitrate as a noninvasive marker of hyperglycemia in type 1 diabetes. P Natl Acad Sci USA, 2007, 104: 15613-15618.

[22] M.A. Kamboures, D.R. Blake, D.M. Cooper, et al., Breath sulfides and pulmonary function in cystic fibrosis. P Natl Acad Sci USA, 2005, 102: 15762-15767.

[23] M. Khoubnasabjafari, V. Jouyban-Gharamaleki, R. Ghanbari, et al., Exhaled breath condensate as a potential specimen for diagnosing COVID-19. Bioanalysis, 2020,
12: 1195-1197.

[24] B. Shan, Y. Y. Broza, W. J. Li, et al., Multiplexed Nanomaterial-Based Sensor Array for Detection of COVID-19 in Exhaled Breath. ACS Nano, 2020, 14: 12125-12132.

[25] Y.S. Chen, Y.X. Zhang, F. Pan, et al., Breath Analysis Based on Surface-Enhanced Raman Scattering Sensors Distinguishes Early and Advanced Gastric Cancer Patients from Healthy Persons. ACS Nano, 2016, 10: 8169-8179.

[26] Y.S. Chen, S.L. Cheng, A.M. Zhang, et al., Salivary Analysis Based on Surface Enhanced Raman Scattering Sensors Distinguishes Early and Advanced Gastric Cancer Patients from Healthy Persons. Journal of Biomedical Nanotechnology, 2018, 14: 1773-1784.

[27] M.A. Aslam, C.L. Xue, Y.S. Chen, et al., Breath analysis based early gastric cancer classification from deep stacked sparse autoencoder neural network. Scientific reports, 2021, 11: 4014.

Copyright $₫$ Cuili Xue, Amin Zhang, Yunsheng Chen, Hui Liang, Jing Tian, Jingpu Zhang, Chen Zhou, Jian Ni, Han Jin, and Daxiang Cui. This is an open-access article distributed under the terms of the Creative Commons Attribution License, which permits unrestricted use, distribution, and reproduction in any medium, provided the original author and source are credited. 